\title{
Major Effective-damping Reduction Achieved by Metallic Coating on YIG Thin Films
}

E. Hermanny ( $\square$ hermanny@cbpf.br)

Centro Brasileiro de Pesquisas Físicas

D. E. González-Chávez

Centro Brasileiro de Pesquisas Físicas

R. L. Sommer

Centro Brasileiro de Pesquisas Físicas

\section{Research Article}

Keywords: Nanomaterials, YIG, Heterostructures, Spin pumping, Damping

Posted Date: July 22nd, 2021

DOI: https://doi.org/10.21203/rs.3.rs-722918/v1

License: (1) This work is licensed under a Creative Commons Attribution 4.0 International License.

Read Full License 
Title

Major effective-damping reduction achieved by metallic coating on YIG thin films

\section{Authors}

E. Hermanny*, D. E. González-Chávez, R. L. Sommer

\section{Affiliation}

Brazilian Center for Physics Research (CBPF), Applied Magnetism Laboratory Rio de Janeiro, Brazil

\section{Corresponding author}

Eric Hermanny, hermanny@cbpf.br

\section{Abstract}

Since its discovery, yttrium iron garnet (YIG) has been object of great interest because of its peculiarly low magnetic damping. Magnetic materials with reduced damping promote microwave power efficiency, longer magnon lifetime and longer spin-wave propagation. Owing to research on charge-to-spin current conversion, the control of magnetic damping in ferromagnetic thin films has recently been achieved by inducing charge current on adjacent metal layer with strong spin-orbit coupling (SOC). We report damping reduction in metallized YIG thin films (YIG/Ag/Ni) without the need of applied charge current and suggest that the origin of this unexpected effect is a strengthening of the FMR uniform mode in the YIG layer, due to frequency- and phase-locking that result from self-synchronization mediated by nonzero spin densities transiting the metallic layers.

\section{Keywords}

Nanomaterials, YIG, Heterostructures, Spin pumping, Damping 


\section{Introduction}

Between 2001 and 2006, the experimental observation of spin pumping ${ }^{[1] ~[2] ~[3] ~ a n d ~ t h e ~ d i r e c t ~}$ ${ }^{[4]}{ }^{[5]}$ and inverse ${ }^{[6]}$ spin Hall effects (SHE and ISHE, respectively) furthered the emergence of spinorbitronics, a trend in spintronic research where pure spin currents and torques originated in spinorbit interactions became the object of intense investigation. One important outcome was transmission of electrical signals by spin-waves travelling in a magnetic insulator ${ }^{[7][8]}$. Another one was full compensation of the damping in a ferromagnet, using charge-to-spin current conversion in adjacent nonmagnetic metallic layers ${ }^{[9]}{ }^{[10]}[11]$. Besides theoretical predictions, the development of new materials has been of utmost importance in creating material conditions to achieve these experimental results. Successful magnon-mediated transmission of signals is challenged by spin relaxation, which renders spin-wave propagation extremely short ranged. YIG is known to be the magnetic material that exhibits by far the lowest magnetic damping. While mastering techniques for the fabrication of YIG thin films is essential to obtain a damping coefficient in the order of $10^{-4}$ to $10^{-}$ ${ }^{5}$, electric current-driven spin injection from a neighboring nonmagnetic metal (NM) was proven to be efficient in artificially reducing the damping in magnetic layers ${ }^{[12] ~[13] ~[14] ~[15] . ~ H o w e v e r, ~ t h e ~}$ threshold current for full compensation is hardly achievable. This limitation has hindered the accomplishment of coherent ferromagnetic-resonance microwave emission, on the path to the predicted realization of the so-called (spin-wave amplification by stimulated emission of radiation) SWASER devices ${ }^{[16]}[17][18][19]$.

Like most garnets, $\mathrm{YIG}\left(\mathrm{Y}_{3} \mathrm{Fe}_{5} \mathrm{O}_{12}\right)$ is characterized by a complex crystalline structure, where 160 atoms are arranged in irregular and slightly twisted tetrahedra, octahedra and dodecahedra inside one pseudocubic unit cell. It is an insulating ferrimagnetic oxide with a bulk damping coefficient of approximately $3 \times 10^{-5}$ at room temperature. Fabrication processes of YIG thin films involve high temperatures (usually post-annealing) to attain crystallization. Surface quality depends on controlled $\mathrm{O}_{2}$ atmosphere during the annealing to prevent stoichiometry 
imbalance due to loss of oxygen and has an impact on the spin mixing conductance of the interface created when a metallic layer is added. In metallized YIG thin films at ferromagnetic resonance (FMR), spin pumping is expected to occur, which can be experimentally evidenced by damping enhancement [2], while spin-orbit coupling-originated spin-to-charge current conversion is evidenced by the detection of voltage in the metallic coating ${ }^{[6][20][21]}$. The interplay between spindiffusion length, spin Hall angle, interfacial spin mixing conductance, layer thickness and, eventually, the presence of magnetic ordering in the metallic layer(s) neighboring the YIG layer will determine the spin-current steady-state regime at ferromagnetic resonance. Literature shows that spin currents may have a strong influence in the coupling of resonating ferromagnetic heterostructures ${ }^{[22][23][24][25]}$ and in the distribution of spin-wave resonance modes ${ }^{[26]}{ }^{[27]}$. Some of our samples exhibited damping reduction, and we have reasons to believe, as detailed below, that the reduction can be explained by this influence.

\section{Methodology}

YIG thin films were deposited on gadolinium gallium garnet (GGG) substrates at room temperature via magnetron sputtering from a 2-inch target and post-annealed in tube furnace within a controlled atmosphere of $\mathrm{O}_{2}$. Prior to deposition, the GGG (111) substrates, with original dimensions $10 \times 10 \times 0.5 \mathrm{~mm}$, were protected with a photoresist coating and mechanically cut into $3 \times 3$ and $3 \times 6 \mathrm{~mm}$ pieces, intended for vector network analysis ferromagnetic resonance (VNAFMR) and spin-to-charge current-conversion measurements $\left(V_{S c}\right)$, respectively. Deposition parameters were: $75 \mathrm{~W}$ rf-power, $12 \mathrm{mTorr}$ work pressure, $15 \mathrm{sccm}$ argon flow and $7.7 \mathrm{~cm}$ targetto-substrate distance. The base pressure was $1.5 \times 10^{-7}$ Torr. The deposition time was calculated to give 55-nm-thick YIG films. The annealing was performed in a controlled $\mathrm{O}_{2}$ atmosphere with a 4hour plateau of $800^{\circ} \mathrm{C}, 5^{\circ} \mathrm{C} / \mathrm{min}$ ramp up and $2^{\circ} \mathrm{C} / \mathrm{min}$ ramp down. After the heat-induced recrystallization, the films were returned to the sputtering chamber for metallic coating. Silver (Ag), copper $(\mathrm{Cu})$, niquel $(\mathrm{Ni})$ and cobalt-iron-boron alloy $\left(\mathrm{Co}_{20} \mathrm{Fe}_{60} \mathrm{~B}_{20}\right)$ were used for single- or double- 
layer coating. The name of each sample, e.g. YIG3 $\operatorname{Ag}(7) \mathrm{Ni}(18)$, describes its layer structure. The number between parenthesis corresponds to the thickness of the layer in nanometers, and the number after "YIG" indicates to which fabrication batch that sample belongs. Whereas the fabrication route was maintained for all batches, there are environmental variants that are impossible to control and may slightly affect the resulting crystal and magnetic qualities of the YIG thin films. Batch classification is thus necessary to avoid compromising the comparative analyses of this study.

The damping of the YIG thin films and the effective damping of each sample were obtained by linear approximation of the frequency dependence of the FMR linewidth. This dependence is modelled by equation:

$$
\Delta H_{H W H M}=\Delta H_{0}+\frac{\alpha}{\gamma} 2 \pi f_{r}
$$

which arise from the solution of the Landau-Lifshitz-Gilbert equation for FMR condition, with small precessional angle approximation ${ }^{[28]}$. The index HWHM indicates that we are using the halfwidth-at-half-maximum convention for the linewidth, $\Delta H_{0}$ is the inhomogeneous line broadening, $\gamma$ is the gyromagnetic ratio and $f_{r}$ is the resonance frequency. All FMR measurements were performed with dc magnetic field applied parallel to the plane of the films.

The FMR linewidth at each frequency, in turn, was calculated from the Lorentzian fit for the corresponding FMR-absorption derivative peak. The Lorentzian behavior of the measured FMR peaks and the linear behavior of the frequency dependence of the linewidth in all the samples ruled out any relevant participation of non-linear relaxation processes, like two-magnon scattering ${ }^{[29]}$, in their magnetization dynamics.

The experimental setup to measure the spin-to-charge current conversion consisted in placing on the metallic surface of the sample two contact probes connected to a nanovoltmeter, while driving the YIG layer to FMR. The obtained voltage $\left(V_{s c}\right)$ was then divided by the electric 
resistance offered by the sample between the two probes. For correct measurement, this experiment must observe the spin-Hall geometry ${ }^{[6]}$, given by the following expression ${ }^{[30]}$, which describes the conversion of spin current $\left(\boldsymbol{J}_{\boldsymbol{s}}\right)$ into charge current $\left(\boldsymbol{J}_{\boldsymbol{c}}\right)$ via spin-orbit coupling:

$$
J_{c}=\theta_{S H} \frac{(-2 e)}{\hbar} J_{s} \times \boldsymbol{\sigma}
$$

where $\theta_{S H}$ is the spin Hall angle, characteristic of the material, $e$ is the electron charge, and $\boldsymbol{\sigma}$ is the spin-polarization unit vector of the spin current.

\section{Results and discussion}

Effective saturation magnetization $\left(4 \pi M_{e f f}\right)$ of 1460 Gauss was obtained with agreed results by vibrating sample magnetometer (VSM) and VNA-FMR techniques (Figures 1a and 1b, respectively). RX-diffraction spectra showed <111> oriented YIG phase (Fig. 1c), with no evidence of the presence of any other phase, and rms roughness as low as $0,12 \mathrm{~nm}$ was calculated from AFM surface images (Fig. 1d). The Gilbert damping coefficient ( $\alpha$ ) of the YIG thin films used in this study was $7.2 \times 10^{-4}, 8.4 \times 10^{-4}, 9.5 \times 10^{-4}$ or $10.0 \times 10^{-4}$, according to the batch to which they belong. These values are compatible with values reported by other groups in the literature describing the use of YIG in spintronics ${ }^{[31]}[32]$. 
(c)

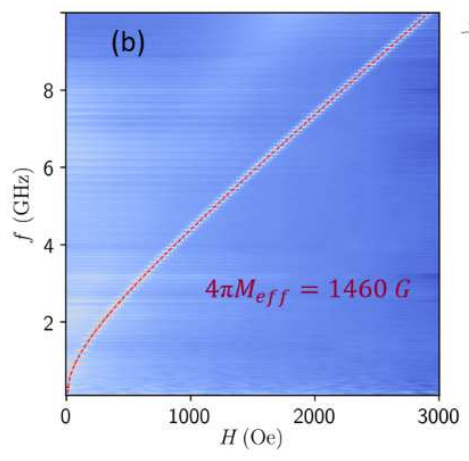

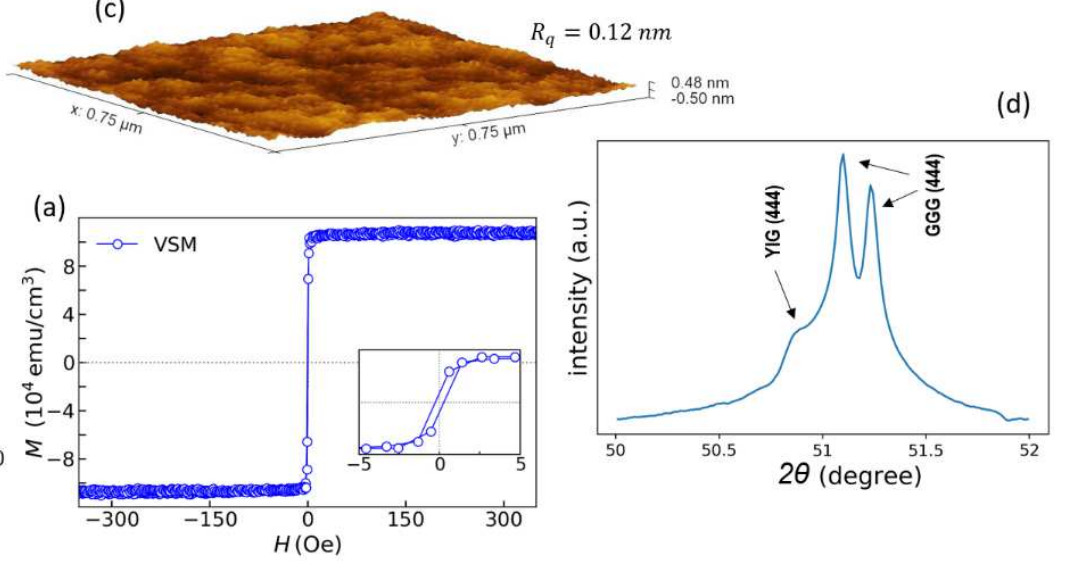

Figure 1: YIG thin-film characterization. (a) Magnetization curve measured with VSM. The inset shows the same curve, amplified in the horizontal axis; (b) Saturation magnetization calculated from the VNA-FMR dispersion curve, where the red dotted line is a fitting curve modelled by the Kittel formula; (c) Surface roughness calculated from AFM image; (d) Identification of the YIG(111) phase via X-ray diffraction.

The finding that is the object of this article originated in our research on spin-to-charge current conversion in ferromagnetic metals, whereby a YIG thin film is driven to FMR in order to inject spins through a NM spacer and into an adjacent ferromagnetic metallic layer. When checking the presence of spin-pumping, we came across an unexpected result for the damping enhancement of one of the samples, YIG1 $\operatorname{Ag}(10) \mathrm{Ni}(20)$, which exhibited, not an enhancement of the damping, but a sharp reduction instead, also accompanied by a narrowing of the FMR linewidths along the entire frequency range (Fig. 2a, Table 1). The signal amplitude of the FMR absorption derivative increased for this metallized sample, as compared with the bare YIG film, while the Lorentzian line fitting was remarkably able to better approach the measured data. The other samples, where either $\mathrm{Ni}$ or $\mathrm{CoFeB}$ was the spin-sink material and either $\mathrm{Ag}$ or $\mathrm{Cu}$ was the spacer, presented damping enhancement as expected (Fig. 2a and 2b, Table 1). 

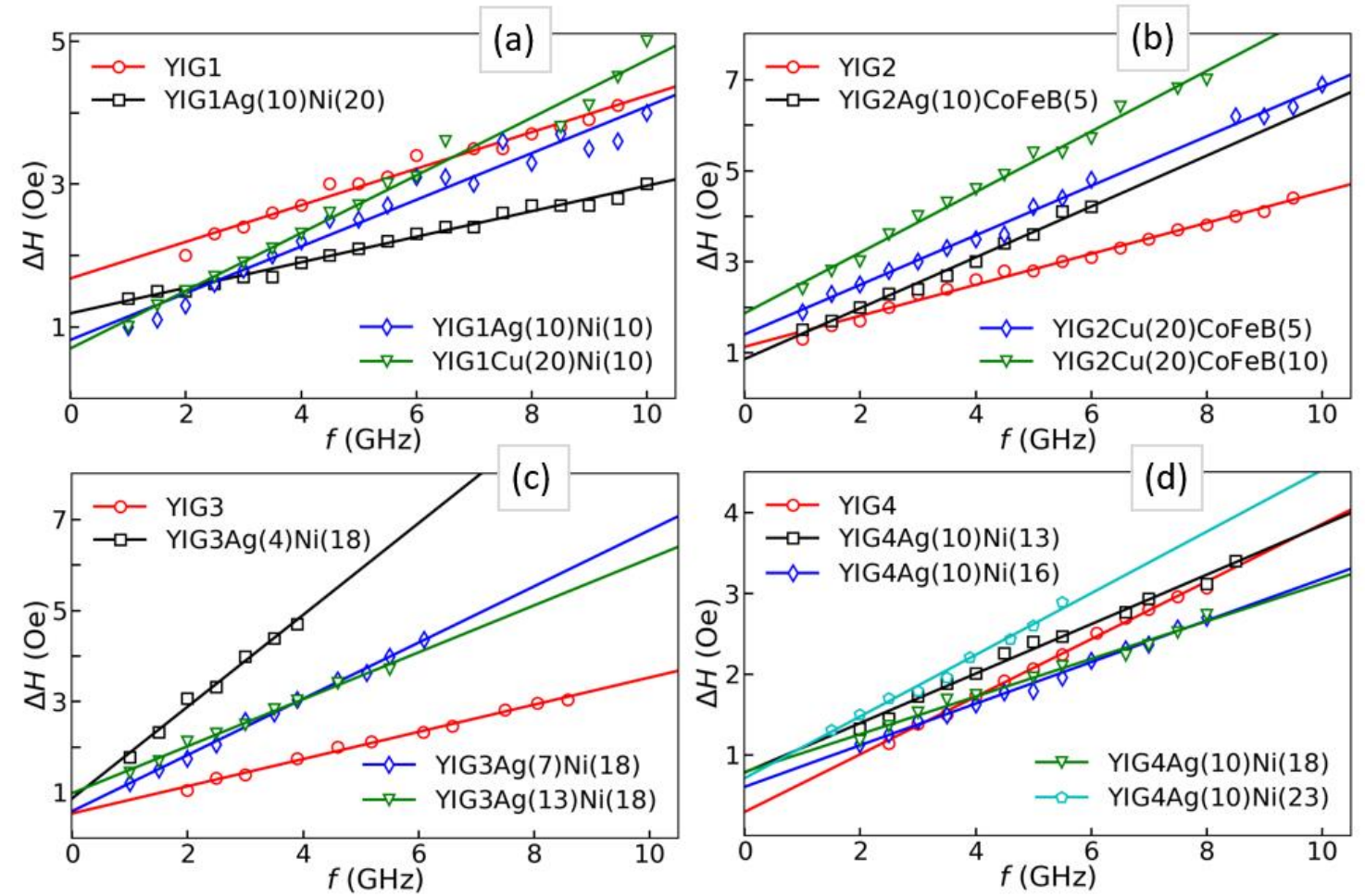

Figure 2: Linear fits. Frequency dependence of the FMR linewidth, for samples belonging to batches YIG1, YIG2, YIG3 and YIG4.

\begin{tabular}{|l|c|c|c|}
\cline { 2 - 4 } \multicolumn{1}{c|}{} & $\boldsymbol{\alpha}\left(10^{-4}\right)$ & $\boldsymbol{\Delta} \boldsymbol{\alpha}\left(10^{-4}\right)$ & $\%$ \\
\hline YIG1 & $7.2 \pm 0.3$ & - & - \\
\hline YIG1Ni(10) & $14.6 \pm 0.5$ & $7.4 \pm 0.8$ & $105 \pm 15$ \\
\hline YIG1Ag(10)Ni(20) & $5.0 \pm 0.1$ & $-2.2 \pm 0.4$ & $-30 \pm 4$ \\
\hline YIG1Ag(10)Ni(10) & $9.2 \pm 0.5$ & $2.0 \pm 0.8$ & $28 \pm 12$ \\
\hline YIG1Cu(20)Ni(10) & $11.3 \pm 0.4$ & $4.1 \pm 0.7$ & $57 \pm 12$ \\
\hline \multicolumn{3}{|c|}{} \\
\hline YIG3 & $8.4 \pm 0.2$ & - & - \\
\hline YIG3Ag(4)Ni(18) & $28.2 \pm 1.3$ & $19.8 \pm 1.5$ & $235 \pm 25$ \\
\hline YIG3Ag(7)Ni(18) & $17.3 \pm 0.4$ & $8.9 \pm 0.6$ & $110 \pm 10$ \\
\hline YIG3Ag(13)Ni(18) & $14.4 \pm 0.5$ & $6.0 \pm 0.7$ & $72 \pm 10$ \\
\hline
\end{tabular}

\begin{tabular}{|l|c|c|c|}
\cline { 2 - 4 } \multicolumn{1}{c|}{} & $\boldsymbol{\alpha}\left(10^{-4}\right)$ & $\boldsymbol{\Delta \alpha}\left(10^{-4}\right)$ & $\boldsymbol{\%}$ \\
\hline YIG2 & $9.5 \pm 0.2$ & - & - \\
\hline YIG2CoFeB(5) & $66.0 \pm 5.7$ & $56.5 \pm 5.9$ & $595 \pm 75$ \\
\hline YIG2 $\mathrm{Ag}(10) \mathrm{CoFeB}(5)$ & $15.2 \pm 0.9$ & $5.7 \pm 1.1$ & $60 \pm 13$ \\
\hline YIG2Cu(20)CoFeB(5) & $15.2 \pm 0.3$ & $5.7 \pm 0.5$ & $62 \pm 8$ \\
\hline YIG2Cu(20)CoFeB(10) & $18.6 \pm 0.5$ & $9.1 \pm 0.7$ & $100 \pm 10$ \\
\hline
\end{tabular}

\begin{tabular}{|l|c|c|c|}
\hline YIG4 & $10.0 \pm 0.2$ & - & - \\
\hline YIG4Ag(10)Ni(13) & $8.6 \pm 0.3$ & $-1.4 \pm 0.5$ & $-14 \pm 5$ \\
\hline YIG4Ag(10)Ni(16) & $7.2 \pm 0.2$ & $-2.8 \pm 0.4$ & $-28 \pm 3$ \\
\hline YIG4Ag(10)Ni(18) & $6.5 \pm 0.3$ & $-3.5 \pm 0.5$ & $-34 \pm 4$ \\
\hline YIG4Ag(10)Ni(23) & $10.7 \pm 0.4$ & $0.7 \pm 0.6$ & $7 \pm 6$ \\
\hline
\end{tabular}

\begin{tabular}{|l|c|c|c|l|l|l|l|l|}
\hline YIG5 & $8.4 \pm 0.2$ & - & - & & YIG5Cu(10) Ni(18) & $13.2 \pm 0.6$ & $4.8 \pm 0.8$ & $57 \pm 11$ \\
\hline
\end{tabular}

Table 1: Damping ( $\alpha$ ) of the samples belonging to batches YIG1, YIG2, YIG3, YIG4 and YIG5. The damping variation $(\Delta \alpha)$ and correspondent percentage change express the enhancement (or reduction) of the damping of metallized samples with respect to the bare YIG sample. 
Narrowing of the linewidth indicates that there has been a decrease in the whole relaxation of the magnetization dynamics, i.e., a reduction in the ensemble of all kinds of relaxation processes, either linear or nonlinear, either local or nonlocal. The reduction in the Gilbert damping coefficient observed for the YIG1 $\mathrm{Ag}(10) \mathrm{Ni}(20)$ sample confirms that the reduction in linewidth is due to a decrease in the relaxation processes that characterize the magnetic system under focus, and not simply due to a decrease in $\Delta H_{0}$.

Pronounced damping enhancement (Table 1) and large $\Delta H_{0}$ (above 4 Oe) of the samples lacking a NM spacer were also noted. According to reports on similar FM/FM structures, these are the result of exchange coupling between the layers due to proximity ${ }^{[31]}$, and of inhomogeneous distribution of the exchange coupling throughout the interface ${ }^{[33]}$, respectively. The use of NM spacers is precisely intended to eliminate static coupling between the FM layers, and because Ag and $\mathrm{Cu}$ have long spin-diffusion length and weak SOC, they permit spin transport between the FM layers, with minimal dissipation ${ }^{[34][31]}$. Now let us observe the extrapolation of the linear fit of the other samples (Fig. 2). The quality of our YIG thin films reflects on the small values for inhomogeneous line broadening $\left(\Delta H_{0}<2\right.$ Oe), which is usually due to defects on the sample but can be influenced by sample dimensions ${ }^{[19]}$. The presence of finite $\Delta H_{0}$ in our samples is probably caused by imperfections originated when the substrates were mechanically cut into the desired size.

Verification samples were fabricated (batches YIG3, YIG4 and YIG5) to consolidate the finding via reproducibility and to check the range of $\mathrm{Ag}$ and $\mathrm{Ni}$ layer thicknesses for which damping reduction is obtained. The results (Figs. 2c and 2d, Table 1) confirm significant damping reduction for samples with Ag layer thickness of $10 \mathrm{~nm}$, and Ni layer thicknesses of 13, 16 and 18 $\mathrm{nm}$. The sharpest reduction $\left(3.5 \times 10^{-4}\right)$ was obtained for the YIG4Ag(10)Ni(18) sample, corresponding to a reduction of at least $30 \%$. With respect to this sample, the only difference of sample $\mathrm{YIG} 5 \mathrm{Cu}(10) \mathrm{Ni}(18)$ is that $\mathrm{Ag}$ is substituted by $\mathrm{Cu}$ in the spacer layer, and damping 
enhancement of $4.8 \times 10^{-4}$ was observed in this case (Table 1), which proves that the silver element plays an essential role in the spacer layer for the achievement of damping reduction.

\section{Energy balance}

Given the complexity of the processes potentially involved, we choose to approach the discussion about the observed damping reduction in YIG/Ag/Ni structures in terms of the energy conservation principle. Figure 3 shows a diagram of the energy balance in the FMR uniform mode.

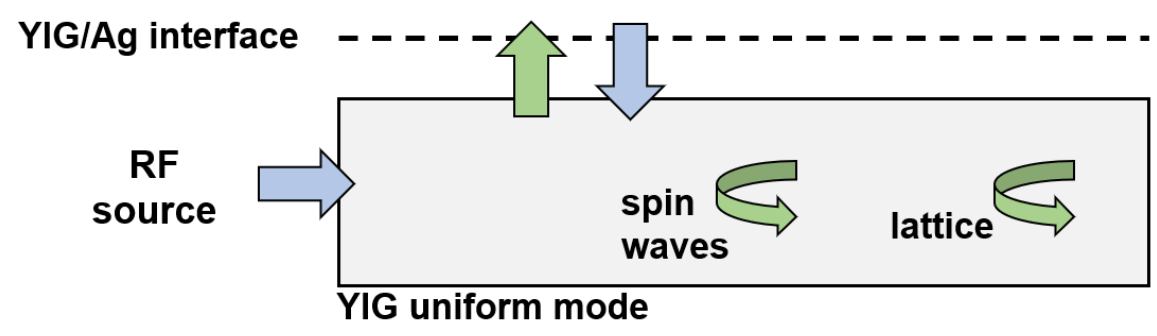

Figure 3: Energy balance. Energy balance of the FMR uniform mode in the YIG layer of a YIG/Ag/Ni sample. The arrows indicate the possible channels for energy entering (blue) or leaving (green) the uniform mode.

The rf power absorbed by the sample is measured by the FMR technique and corresponds to the power absorbed by the uniform mode. The absorption line obtained by scanning the dc magnetic field near the resonance condition gives substantial information about the relaxation mechanisms whereby energy abandons the uniform mode [35]. Energy enters the uniform mode of the YIG layer coming either from the rf source, through interaction with the rf field, or from the YIG/Ag interface, via spin-transfer torque (STT). On the other hand, the uniform mode can lose energy either to the YIG lattice, by coupling with lattice vibrations (magnon-phonon interactions) ${ }^{[36]}$, to other resonance modes $(k \neq 0)$, by coupling with spin-wave modes (magnon-magnon interactions) ${ }^{[37]}$, or through the YIG/Ag interface, by transmitting angular momentum via spin pumping ${ }^{[3]}$. It should be 
noted that, while direct interactions of the uniform mode with the lattice contribute linearly with frequency to the linewidth, the ones mediated by spin waves are nonlinear ${ }^{[28]}$.

From the point of view of the energy balance of the FMR uniform mode in the YIG layer at dynamic equilibrium, viabilities for damping reduction can be investigated by the following considerations:

1. Spin injection from the Ni layer, through the Ag spacer and into the YIG layer is possible when overlapping of the YIG and Ni dispersion curves permits concurrent resonance. Their dispersions curves do overlap at low frequencies, but they separate as the frequency raises, which means that spin injection from the Ni layer would be in contradiction with the linear behavior observed for the FMR linewidths;

2. Spin injection into the YIG layer resulting from SHE in the Ag layer or REE on the interfaces is ruled out because there is no applied voltage generating charge current to justify input of energy into the YIG layer through spin-Hall or Rashba-Edelstein charge-tospin current conversion;

3. If for any reason the ability of the YIG to absorb rf power were modified by the added metallic layers, this would affect the intensity of the absorption peak, but would not reduce the damping;

4. The above considerations imply that the observed damping reduction must be the result of the inhibition of local relaxation processes within the YIG layer. Furthermore, as only Gilbert-type damping is observed, nonlinear relaxation processes are not relevant in the magnetization dynamics in the YIG layer or on the YIG/Ag interface (for our samples). Therefore, inhibition of direct interactions of the uniform mode with the lattice must be the cause of the observed damping reduction. 


\section{Dynamic magnetic coupling}

In a seminal article [38] called Dynamic Exchange Coupling in Magnetic Bilayers (2003), B. Heinrich, Y. Tserkovnyak, G. Woltersdorf, A. Brataas, R. Urban and G. E. W. Bauer announced a new coupling mechanism between resonating magnetic layers, mediated by pure spin currents flowing from each magnetic layer to the other, through a nonmagnetic-metal spacer. One remarkable feature of this study is that the coupling between the magnetic layers occurs because the compensating spin currents synchronize, and the determinant condition for this synchronization is that the separately controlled magnetizations of the layers are oriented into alignment. The interest in dynamic coupling was carried over to spin-torque nano-oscillators (STNOs) and the

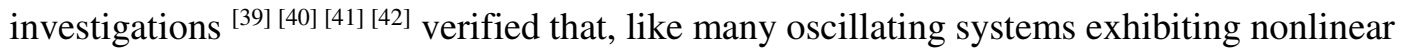
interactions, STNOs tend to frequency-lock when the difference between their resonance frequencies is small enough. The frequency locking for two coupled STNOs is characterized by a narrowing of the locked-frequency signal linewidth compared with the linewidths of the separately resonating STNOs. Frequency locking is usually accompanied by phase-locking into a single resonance, whereby an increase in power is also verified. The coupling strength can bring together in frequency two resonances that would otherwise be resonating in different frequencies. Frequency jumps due to frequency locking in STNOs are reported by experimental and theoretical studies to range from about 10 to $100 \mathrm{MHz}$.

These findings imply that STTs can drive independent magnetizations into coupled dynamics ${ }^{[39]}$. Investigations of magnetization dynamics in STNOs closely relate to those in ferromagnetic resonance because STT is the Onsager reciprocal of spin pumping ${ }^{[43]}$. Thus, in our device, spin currents in the Ag layer and/or spin accumulation on the YIG/Ag interface could intermediate dynamic coupling of the YIG and the Ni layers, or even a self-synchronization of the uniform-mode magnons in YIG, as described in the next section. This coupling will strengthen the resonance mode at which it occurs. Magnon-phonon coupling, which is substantially responsible for the relaxation in ferromagnetic insulators, will now have to compete with the spin current-driven 
dynamic coupling. This strengthening of the uniform mode will inhibit the relaxation due to coupling with lattice vibrations (phonons) and will result in a reduction of damping in the garnet.

\section{Self-synchronization of the uniform mode}

Upon the YIG thin-film fabrication on the GGG substrate, all attempts are usually made to obtain a monocrystalline thin film. The division of the YIG thin film into separate crystalline phases is usually undesirable. When this is the case, FMR measurements exhibit double or multiple peaks with slightly different resonance fields, each corresponding to a different crystalline phase. This is explained by the fact that each of these phases has a slightly different saturation magnetization, which reflects on a slightly shifted FMR dispersion curve for each phase ${ }^{[32]}$. At $9.5 \mathrm{GHz}$, the presence of a small secondary peak in the FMR line shape of our bare YIG1 thin film contrasts with the line shape of the YIG1 $\operatorname{Ag}(10) \mathrm{Ni}(20)$ sample, which exhibits a perfect single Lorentzian peak (Fig. 4). This is evidence of the strengthening of the uniform mode in the YIG layer. The precessing magnetizations of the YIG crystalline phases with slightly different natural resonance frequencies become frequency-locked due to a coupling spin torque ${ }^{[39]}$. Another evidence of this coupling is that the power absorbed by the metallized YIG thin film is at least twice as high as the bare YIG. It should be noted that self-synchronization of oscillating magnetizations has been extensively reported to occur via spin torques originating in spin waves, electric current, microwave field or dipole coupling (see Ref. ${ }^{[44]}$ and references therein), whereas the coupling torque elicited in the theoretical treatment, as developed in Ref. ${ }^{[39]}$, of the dynamic coupling driven by pure spin currents gives a much stronger coupling as compared with the coupling driven by spin-polarized electric currents ${ }^{[44]}$. 

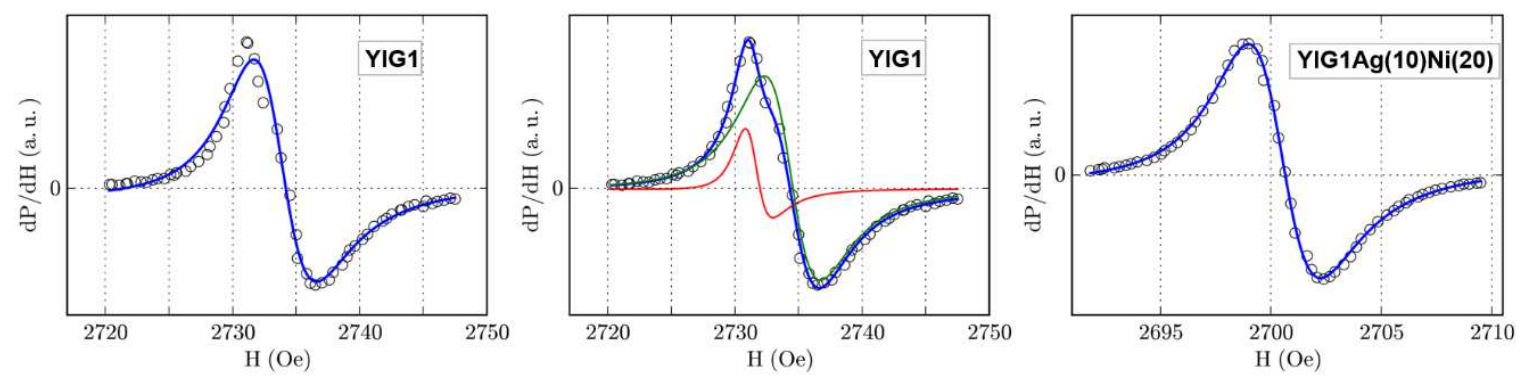

Figure 4: Lorentzian resonance lines. Comparison of the FMR line shapes at $9.5 \mathrm{GHz}$ of YIG without and with metallic coating. On the left and on the right, single-peak Lorentzian fits (blue line) to the measured data (black circles). In the center, a two-peak Lorentzian fit, where the blue line is the sum of the red and the green lines. Note that the FMR absorption-line graph of the metallized YIG (on the right) has a different horizontal scale for the applied field $\mathrm{H}$ from the graphs of the bare YIG thin film.
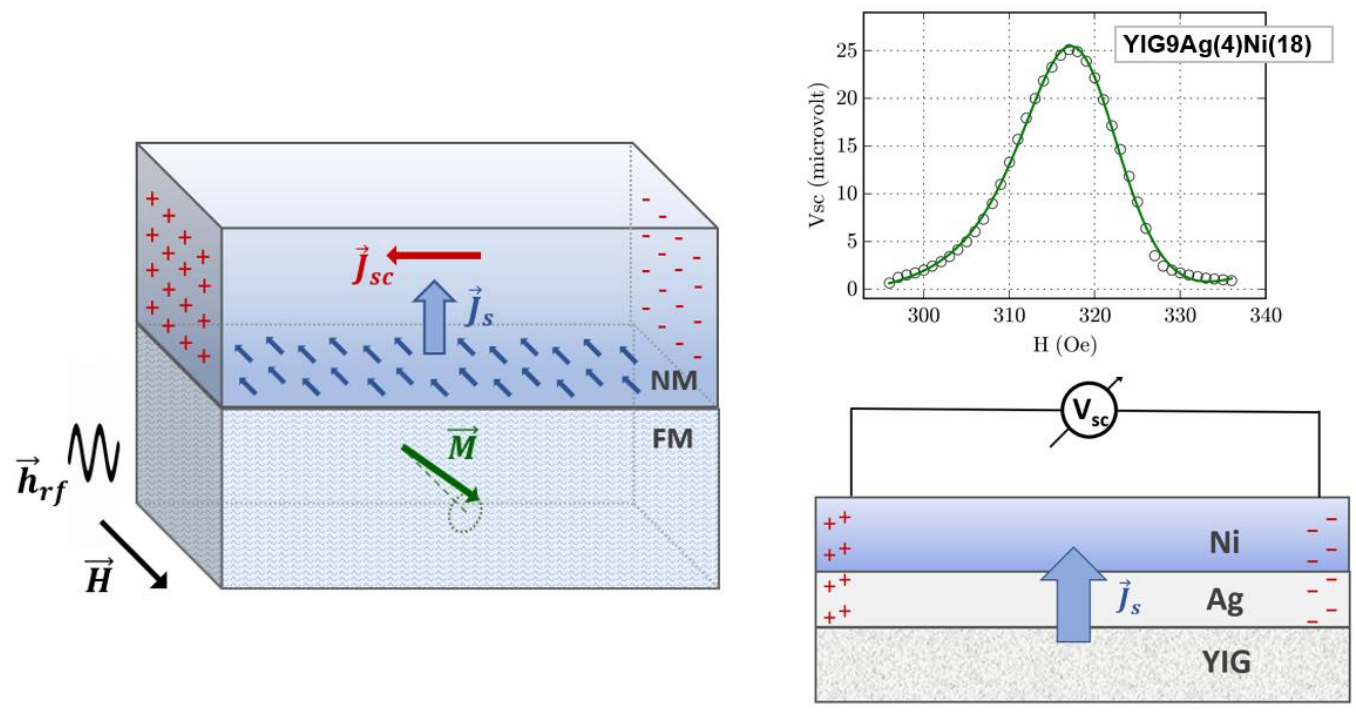

Figure 5: Spin-current detection. The diagram on the left represents the dynamics of spin pumping and ISHE in a FM/NM structure, where the NM has strong SOC. The small arrows represent the polarization $\sigma$ of the spins accumulated at the interface. The spin-Hall geometry reflects the relation described in Equation 2. Below: Detection of the spin-to-charge current conversion in our trilayer structure, where YIG is the spin injector, Ag is the spacer and Ni, the spin sink. Above: The measured voltage $\left(V_{s c}\right)$ results from spin-to-charge current conversion. The green line is the Lorentzian fit to the measured data (black circles). 


\begin{tabular}{|l|c|c|}
\hline & $\Delta \alpha\left(10^{-4}\right)$ & $I_{s c}=V_{s c} / R(\mu \mathrm{A})$ \\
\hline YIG1 Ag(10)Ni(20) & -2.2 & 0.04 \\
\hline YIG1Cu(20)Ni(10) & 4 & 0.42 \\
\hline YIG3Ag(4) Ni(18) & 19.8 & 0.18 \\
\hline YIG3Ag(7) Ni(18) & 8.9 & 0.09 \\
\hline YIG4Ag(10)Ni(13) & -1.4 & 0.01 \\
\hline YIG4Ag(10)Ni(16) & -2.8 & 0.06 \\
\hline YIG4Ag(10)Ni(18) & -3.5 & 0.02 \\
\hline YIG4Ag(10)Ni(23) & 0.7 & 0.04 \\
\hline YIG5Cu(10) Ni(18) & 4.8 & 0.28 \\
\hline YIG2Ag(10)CoFeB(5) & 6.1 & 0.02 \\
\hline YIG2Cu(20)CoFeB(10) & 9.1 & 0.05 \\
\hline
\end{tabular}

Table 2: Spin-to-charge current conversion $\left(I_{s c}\right)$, calculated from $V_{S c}$ and the resistance $(R)$ measured between two contact probes placed on the metallic surface of each sample.

\section{The transport of nonzero spin densities}

Although the exact regime of pure spin currents and non-equilibrium spin accumulation (nonzero spin density) throughout the $\mathrm{YIG1Ag}(10) \mathrm{Ni}(20)$ sample at FMR remains unclear, the presence of spin current is evidenced via detection of spin current-induced voltage on the metallic Ni surface, using the spin-Hall geometry (Fig. 5, Table 2). Both ISHE and inverse Rashba-Edelstein effect (IREE) ${ }^{[45]}$ can be involved in the spin-to-charge current conversion that generates the observed voltage ${ }^{[46][20]}[47]$, even though the presence of ferromagnetism on the interfaces renders uncertain the participation of IRRE ${ }^{[20]}$. The spin current reaching the $\mathrm{Ag} / \mathrm{Ni}$ interface will be partially reflected and partially transmitted into the Ni layer, where it will be converted into charge current via ISHE and via anomalous spin Hall effect (ASHE) ${ }^{[21]}$. Part of the spin current might also be converted into charge current at the interface via IREE. These effects observe similar spatial geometry and contribute to the spin-to-charge current conversion sensed as the $V_{s c}$ voltage.

While a similar damping reduction (relative to the bare magnetic film at FMR, and without applied current) reported in Ref. ${ }^{[48]}$ for a magnetic ultrathin-film structure was mainly attributed 
to Rashba spin-orbit torques generated at backflow of spin current, we propose that backflow of spin current on the $\mathrm{Ag} / \mathrm{Ni}$ interface is essential for the establishment of the above-mentioned dynamic magnetic coupling in our sample. Thus, in the FMR condition, the precession magnetization of the YIG pumps spin angular momentum through the YIG/Ag interface, then polarized spins accumulate near the interface and diffuse across the Ag layer with virtually no spin-flip losses ${ }^{[34]}$ until they reach the $\mathrm{Ag} / \mathrm{Ni}$ interface, which (partially) reflects this spin current back to the Ag layer towards and through the YIG/Ag interface, into the YIG layer ${ }^{[22]}$. When we compare the intensity of $I_{S c}$ with the amount of damping reduction obtained for the four samples (Table 2, in red), the relation between them remains unclear. But when we compare the intensities of $I_{S c}$ for these samples with the intensities for corresponding copper-substituted

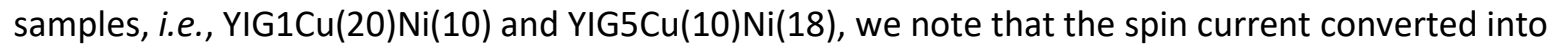
charge current in the latter case (Cu spacer) is one order of magnitude above, as compared to the former case (Ag spacer). This difference is in accordance with the assumption that backflow of spin current at the $\mathrm{Ag} / \mathrm{Ni}$ interface is involved in the observed damping reduction, and consequently also supports the involvement of dynamic coupling. This picture is consolidated by the realization that backflow of spin current from the $\mathrm{Ag} / \mathrm{Ni}$ interface and across the $\mathrm{Ag}$ spacer will at least partly traverse the YIG/Ag interface back into the YIG layer, and thus compensate the outflow of angular momentum caused by spin pumping. After all, in the frame of the uniform mode's energy balance

(Fig. 3), the inhibition of local damping in the YIG must overcome the non-local damping caused by the spin-pumping so that the damping-reduction effect can take place.

\section{The role of the Ni layer and one unanswered question}

Nickel can also be excited into FMR, and concurrent excitation of YIG and nickel is possible at low frequencies. However, as reasoned above, concurrent excitation of the Ni layer is 
not likely to play a relevant role in the observed damping reduction. Besides the linear frequency dependence of the linewidth, the results obtained for the YIG4Ag(10)Ni(x) verification samples also support this assumption. Three samples with different thicknesses of Ni layer exhibit damping reduction. While spin-pumping efficiency is expected to be inversely proportional to injector-layer thickness ${ }^{[19]}$, the observed damping-reduction effect increases for thicker layers of Ni. In contrast to Ni-layer thickness, the reduction effect seems to be extremely sensitive to Ag-layer thickness. Among the unanswered questions that this study elicits is why the samples with Ag layer thickness different from $10 \mathrm{~nm}$ do not exhibit damping reduction.

\section{Conclusion}

This article announces the achievement of major damping reduction, of at least $30 \%$, in YIG thin films by metallization, without the need of applied current. The discovery is unprecedented, either for YIG or any other magnetic material, and is consolidated by reproducibility. Although the theory behind the observed effect of the Ag/Ni metallization on YIG remains unclear, there is strong evidence that spin current-driven self-synchronization of the FMR uniform mode in the YIG layer plays a decisive role. Further investigation involving poli- and monocrystalline YIG, greater range of layer thicknesses and applied charge current is necessary to determine the impact this finding may have on the development of new magnonic and spintronic devices.

\section{Data availability}

The datasets generated during and/or analyzed during the current study are available from the corresponding author on reasonable request. 


\section{References}

[1] Mizukami, S., Ando, Y., and Miyazaki, T. The study on ferromagnetic resonance linewidth for NM/80NiFe/NM (NM = Cu, Ta, Pd and Pt) Films. Jpn. J. Appl. Phys. 40, 580-585 (2001).

[2] Tserkovnyak, Y., Brataas, A. and Bauer, G. E. Enhanced Gilbert damping in thin ferromagnetic films. Phys. Rev. Lett. 88, 117601 (2002).

[3] Tserkovnyak, Y., Brataas, A. and Bauer, G. E. Spin pumping and magnetization dynamics in metallic multilayers. Phys. Rev. B 66, 224403 (2002).

[4] Kato, Y. K., Myers, R. C., Gossard, A. C. and Awschalom, D. D. Observation of the spin Hall effect in semiconductors. Science 306, 1910-1913 (2004).

[5] Wunderlich, J., Kaestner, B., Sinova, J. and Jungwirth, T. Experimental observation of the spin-Hall effect in a two-dimensional spin-orbit coupled semiconductor system. Physical Review B 94, 047204 (2005).

[6] Saitoh, E., Ueda, M., Miyajima, H. and Tatara, G. Conversion of spin current into charge current at room temperature: Inverse spin-Hall effect. App. Phys. Lett. 88, 182509, (2006).

[7] Kajiwara, Y. et al. Transmission of electrical signals by spin-wave interconversion in a magnetic insulator. Nat. Lett. 464, 262-267, (2010).

[8] Cornelissen, L. J., Liu, J., Duine, R. A., Youssef, J. B. and van Wees, B. J. Long-distance transport of magnon spin information in a magnetic insulator at room temperature. Nat. Phys. Lett. 11, 1022-1026, (2015).

[9] Demidov, V. E. et al. Magnetic nano-oscillator driven by pure spin current. Nat. Mat. Lett. 11, 1028-1031, (2012).

[10] Hamadeh, A. et al. Full control of the spin-wave damping in a magnetic insulator using spinorbit torque. Phys. Rev. Lett. 113, 197203, (2014).

[11] Wimmer, T. et al. Spin transport in a magnetic insulator with zero effective damping. Phys. Rev. Lett. 123, 257201, (2019).

[12] Wang, Z., Sun, Y., Wu, M., Tiberkevich, V. and Slavin, A. Control of spin waves in a thin film ferromagnetic Insulator through interfacial spin scattering. Phys. Rev. Lett. 107, 146602, (2011).

[13] Haidar, M. et al. Controlling Gilbert damping in a YIG film using nonlocal spin currents. Phys. Rev. B 94, 180409 (R), (2016).

[14] Tiwari, D. et al. Antidamping spin-orbit torques in epitaxial-Py(100)/ $\beta$-Ta. Appl. Phys. Lett. 111, 232407, (2017). 
[15] Evelt, M. et al. High-efficiency control of spin-wave propagation in ultra-thin yttrium iron garnet by the spin-orbit torque. Appl. Phys. Lett. 108, 172406, (2016).

[16] Berger, L. Emission of spin waves by a magnetic multilayer traversed by a current. Phys. Rev. $B$ 54, 9353-9358, (1996).

[17] Berger, L. Multilayers as spin-wave emitting diodes. J. Appl. Phys. 81, 4880-4882, (1997).

[18] Berger, L. Spin-wave emitting diodes and spin diffusion in magnetic multilayers. IEEE Trans. Magn. 34, 3837-3841, (1998).

[19] Collet, M. et al. Generation of coherent spin-wave modes in yttrium iron garnet microdiscs by spin-orbit torque. Nat. Commun. 7, 10377, (2016).

[20] Rojas-Sánchez, J. C. et al. Spin-to-charge conversion using Rashba coupling at the interface between non-magnetic materials. Nat. Commun. 4, 2944, (2013).

[21] Das, K. S., Schoemaker, W. Y., van Wees, B. J. and Vera-Marun, I. J. Spin injection and detection via the anomalous spin Hall effect of a ferromagnetic metal. Phys. Rev. B 96, 220408, (2017).

[22] Tserkovnyak, Y., Brataas, A. and Bauer, G. E. Dynamic exchange coupling and Gilbert damping in magnetic multilayers. J. Appl. Phys. 93, 7534-7538, (2003).

[23] Arena, D. A., Vescovo, E., Kao, C.-C., Guan, Y. and Bailey, W. E. Weakly coupled motion of individual layers in ferromagnetic resonance. Phys. Rev. B 74, 064409, (2006).

[24] Skarsvag, H., Bauer, G. E. W. and Brataas, A. Current-induced magnetization dynamics in two magnetic insulators separated by a normal metal. Phys. Rev. B 90, 054401, (2014).

[25] Gladczuk, Ł. et al. Spin-current mediated exchange coupling in MgO-based magnetic tunnel junctions. Phys. Rev. B 103, 064416, (2021).

[26] Demidov, V. E. et al. Control of magnetic fluctuations by spin current. Phys. Rev. Lett. 107, 107204, (2011).

[27] Skarsvag, H., Kapelrud, A. and Brataas, A. Spin waves in ferromagnetic insulators coupled via a normal metal. Phys. Rev. B 90, 094418, (2014).

[28] Zakeri, K. et al. Spin dynamics in ferromagnets: Gilbert damping and two-magnon scattering. Phys. Rev. B 76, 104416, (2007).

[29] Lindner, J. et al. Non-Gilbert-type damping of the magnetic relaxation in ultrathin ferromagnets: Importance of magnon-magnon scattering. Phys. Rev. B 68, 060102, (2003).

[30] Althammer, M. Pure spin currents in magnetically ordered insulator/normal metal heterostructures. J. Phys. D 51, 313001, (2018). 
[31] Wang, H., Du, C., Hammel, P. C. and Yang, F. Spin current and inverse spin Hall effect in ferromagnetic metals probed by Y3Fe5O12-based spin pumping. Appl. Phys. Lett. 104, 202405, (2014).

[32] Heinrich, B. et al. Spin pumping at the magnetic insulator (YIG)/normal metal (Au) interfaces. Phys. Rev. Lett. 107, 066604, (2011).

[33] Li, M. et al. Giant damping enhancement induced by exchange coupling in Y3Fe5O12/Co2FeA10.5Si0.5 bilayers. J. All. Com. 767, 398-402, (2018).

[34] Kardasz, B., Heinrich, O. M. B., Liu, Z. and Freeman, M. Spin current studies in Fe/Ag, $\mathrm{Au} / \mathrm{Fe}$ by ferromagnetic resonance and time-resolved magneto-optics. J. Appl. Phys. 103, 07C509, (2008).

[35] Vonsovskii, S. V. Magnetic resonance in ferromagnetics, in Ferromagnetic Resonance, Pergamon Press, (1966).

[36] Azzawi, S., Hindmarch, A. T. and Atkinson, D. Magnetic damping phenomena in ferromagnetic thin-films and multilayers. J. Phys. D 50, 473001, (2017).

[37] Fletcher, R. C., LeCraw, R. C. and Spencer, E. G. Electron spin relaxation in ferromagnetic insulators. Phys. Rev. 117, 995-963, (1960).

[38] Heinrich, B. et al. Dynamic exchange coupling in magnetic bilayers. Phys. Rev. Lett. 90, 187601, (2003).

[39] Taniguchi, T. Spin-current driven spontaneous coupling of ferromagnets. Phys. Rev. B 98, 104417, (2018).

[40] Mancoff, F. B., Rizzo, N. D., Engel, B. N. and Tehrani, S. Phase-locking in double-pointcontact spin-transfer devices. Nat. Lett. 437, 04036, (2005).

[41] Kaka, S. et al. Mutual phase-locking of microwave spin torque nano-oscillators. Nat. Lett. 437, 04035, (2005).

[42] Rippard, W. H., Pufall, M. R., Kaka, S., Silva, T. J. and Russek, S. E. Injection locking and phase control of spin transfer nano-oscillators. Phys. Rev. Lett. 95, 067203, (2005).

[43] Brataas, A., Tserkovnyak, Y., Bauer, G. E. W. and Kelly, P. J. Spin pumping and spin transfer, in Spin Current, Oxford University Press, pp. 87-135, (2012).

[44] Taniguchi, T. Dynamic coupling of ferromagnets via spin Hall magnetoresistance. Phys. Rev. B 95, 104426, (2017).

[45] Edelstein, V. M. Spin polarization of conduction electrons induced by electric current in twodimensional asymmetric electron systems. Solid State Commun. 73, 233-235, (1990). 
[46] Allen, G., Manipatruni, S., Nikonov, D. E., Doczy, M. and Young, I. A. Experimental demonstration of the coexistence of spin Hall and Rashba effects in $\beta$-tantalum/ferromagnet bilayers. Phys. Rev. B 91, 144412, (2011).

[47] Fan, X. et al. Quantifying interface and bulk contributions to spin-orbit torque in magnetic bilayers. Nat. Commun. 5, 3042, (2014).

[48] Behera, N., Chaudhary, S. and Pandya, D. K. Anomalous anti-damping in sputtered $\beta-T a / P y$ bilayer system. Sci. Rep. 6, 19488, (2016).

\section{Acknowledgements}

The authors acknowledge the Brazilian funding agencies CAPES, CNPq, FAPERJ and FINEP.

\section{Author contribution statements}

E. H. identified the unusual behavior, designed the study, fabricated samples, performed experiments, analyzed and interpreted output data and wrote the manuscript. D. E. G.-C. designed experiments, wrote code, gave technical support and conceptual advice, and revised the manuscript. R. L. S. organized the lab and research funding, revised the manuscript and supervised the project. All authors discussed the results and implications and commented on the manuscript at all stages.

\section{Additional information}

The authors declare the existence of a pending patent application that may imply a financial competing interest in the present article.

Patent applicant: CBPF

Name of inventors: Eric Hermanny, Diego Ernesto González-Chávez, Rubem Luis Sommer Application number: BR 1020210101423

Status of application: pending Specific aspect of manuscript covered in patent application: Method to reduce damping in YIG thin films, consisting in $\mathrm{Ag} / \mathrm{Ni}$ coating. 


\section{Figure legends}

Figure 1: YIG thin-film characterization. (a) Saturation magnetization calculated from the VNAFMR dispersion curve, where the red dotted line is a fitting curve modelled by the Kittel formula;

(b) Surface roughness calculated from AFM image; (c) Identification of the YIG(111) phase via Xray diffraction; (d) Magnetization curve measured with VSM. The inset shows the same curve, amplified in the horizontal axis.

Figure 2: Linear fits. Frequency dependence of the FMR linewidth, for samples belonging to batches YIG1, YIG2, YIG3 and YIG4.

Figure 3: Energy balance. Energy balance of the FMR uniform mode in the YIG layer of a YIG/Ag/Ni sample. The arrows indicate the possible channels for energy entering (blue) or leaving (green) the uniform mode.

Figure 4: Lorentzian resonance lines. Comparison of the FMR line shapes at $9.5 \mathrm{GHz}$ of YIG without and with metallic coating. On the left and on the right, single-peak Lorentzian fits (blue line) to the measured data (black circles). In the center, a two-peak Lorentzian fit, where the blue line is the sum of the red and the green lines. Note that the FMR absorption-line graph of the metallized YIG (on the right) has a different horizontal scale for the applied field $\mathrm{H}$ from the graphs of the bare YIG thin film.

Figure 5: Spin-current detection. The diagram on the left represents the dynamics of spin pumping and ISHE in a FM/NM structure, where the NM has strong SOC. The small arrows represent the polarization $\boldsymbol{\sigma}$ of the spins accumulated at the interface. The spin-Hall geometry reflects the relation described in Equation 2. Below: Detection of the spin-to-charge current conversion in our trilayer structure, where YIG is the spin injector, Ag is the spacer and $\mathrm{Ni}$, the spin sink. Above: The measured voltage $(V S c)$ results from spin-to-charge current conversion. The green line is the Lorentzian fit to the measured data (black circles).

Tables

\begin{tabular}{|c|c|c|c|c|c|c|c|}
\hline & $\boldsymbol{\alpha}\left(10^{-4}\right)$ & $\Delta \alpha\left(10^{-4}\right)$ & $\%$ & & $\boldsymbol{\alpha}\left(10^{-4}\right)$ & $\Delta \alpha\left(10^{-4}\right)$ & $\%$ \\
\hline YIG1 & $7.2 \pm 0.3$ & - & - & YIG2 & $9.5 \pm 0.2$ & - & - \\
\hline YIG1Ni(10) & $14.6 \pm 0.5$ & $7.4 \pm 0.8$ & $105 \pm 15$ & YIG2CoFeB(5) & $66.0 \pm 5.7$ & $56.5 \pm 5.9$ & $595 \pm 75$ \\
\hline YIG1 Ag(10)Ni(20) & $5.0 \pm 0.1$ & $-2.2 \pm 0.4$ & $-30 \pm 4$ & YIG2Ag(10)CoFeB(5) & $15.2 \pm 0.9$ & $5.7 \pm 1.1$ & $60 \pm 13$ \\
\hline YIG1Ag(10)Ni(10) & $9.2 \pm 0.5$ & $2.0 \pm 0.8$ & $28 \pm 12$ & $\mathrm{YIG} 2 \mathrm{Cu}(20) \mathrm{CoFeB}(5)$ & $15.2 \pm 0.3$ & $5.7 \pm 0.5$ & $62 \pm 8$ \\
\hline YIG1Cu(20)Ni(10) & $11.3 \pm 0.4$ & $4.1 \pm 0.7$ & $57 \pm 12$ & YIG2Cu(20)CoFeB(10) & $18.6 \pm 0.5$ & $9.1 \pm 0.7$ & $100 \pm 10$ \\
\hline YIG3 & $8.4 \pm 0.2$ & - & - & YIG4 & $10.0 \pm 0.2$ & - & - \\
\hline YIG3Ag(4)Ni(18) & $28.2 \pm 1.3$ & $19.8 \pm 1.5$ & $235 \pm 25$ & YIG4Ag(10)Ni(13) & $8.6 \pm 0.3$ & $-1.4 \pm 0.5$ & $-14 \pm 5$ \\
\hline YIG3Ag(7)Ni(18) & $17.3 \pm 0.4$ & $8.9 \pm 0.6$ & $110 \pm 10$ & YIG4Ag(10)Ni(16) & $7.2 \pm 0.2$ & $-2.8 \pm 0.4$ & $-28 \pm 3$ \\
\hline YIG3Ag(13)Ni(18) & $14.4 \pm 0.5$ & $6.0 \pm 0.7$ & $72 \pm 10$ & YIG4Ag(10)Ni(18) & $6.5 \pm 0.3$ & $-3.5 \pm 0.5$ & $-34 \pm 4$ \\
\hline & & & & YIG4Ag(10)Ni(23) & $10.7 \pm 0.4$ & $0.7 \pm 0.6$ & $7 \pm 6$ \\
\hline YIG5 & $8.4 \pm 0.2$ & - & - & YIG5Cu(10)Ni(18) & $13.2 \pm 0.6$ & $4.8 \pm 0.8$ & $57 \pm 11$ \\
\hline
\end{tabular}

Table 3: Damping ( $\alpha$ ) of the samples belonging to batches YIG1, YIG2, YIG3, YIG4 and YIG5. The damping variation $(\Delta \alpha)$ and correspondent percentage change express the enhancement (or reduction) of the damping of metallized samples with respect to the bare YIG sample. 


\begin{tabular}{|l|c|c|}
\hline & $\Delta \alpha\left(10^{-4}\right)$ & $I_{s c}=V_{s c} / R(\mu \mathrm{A})$ \\
\hline YIG1 $\mathrm{Ag}(10) \mathrm{Ni}(20)$ & -2.2 & 0.04 \\
\hline $\mathrm{YIG} 1 \mathrm{Cu}(20) \mathrm{Ni}(10)$ & 4 & 0.42 \\
\hline $\mathrm{YIG} 3 \mathrm{Ag}(4) \mathrm{Ni}(18)$ & 19.8 & 0.18 \\
\hline YIG3Ag(7)Ni(18) & 8.9 & 0.09 \\
\hline YIG4Ag(10)Ni(13) & -1.4 & 0.01 \\
\hline YIG4Ag(10)Ni(16) & -2.8 & 0.06 \\
\hline YIG4Ag(10)Ni(18) & -3.5 & 0.02 \\
\hline YIG4Ag(10)Ni(23) & 0.7 & 0.04 \\
\hline YIG5Cu(10)Ni(18) & 4.8 & 0.28 \\
\hline YIG2Ag(10)CoFeB(5) & 6.1 & 0.02 \\
\hline YIG2Cu(20)CoFeB(10) & 9.1 & 0.05 \\
\hline
\end{tabular}

Table 4: Spin-to-charge current conversion $\left(I_{s c}\right)$, calculated from $V_{s c}$ and the resistance $(R)$ measured between two contact probes placed on the metallic surface of each sample.

\section{Authors' contacts}

Eric Hermanny, hermanny@ cbpf.br (corresponding author)

Diego Ernesto González-Chávez, diegogch@cbpf.br

Rubem Luis Sommer, sommer@cbpf.br 